\title{
Delayed-onset rhabdomyolysis after intense exercise
}

\author{
Mathew Kiberd MD, Sam Campbell MB BCh
}

Competing interests: None declared.

This article has been peer reviewed.

Affiliations: From the Department of Anesthesia (Kiberd), Queen Elizabeth II Health Sciences Centre; and the Department of Emergency Medicine (Campbell), Dalhousie University, Halifax, NS

\section{Correspondence to:} Dr. Mathew Kiberd, mathew.kiberd@dal.ca

CMAJ 2011. DOI:10.1503 /cmaj.100210

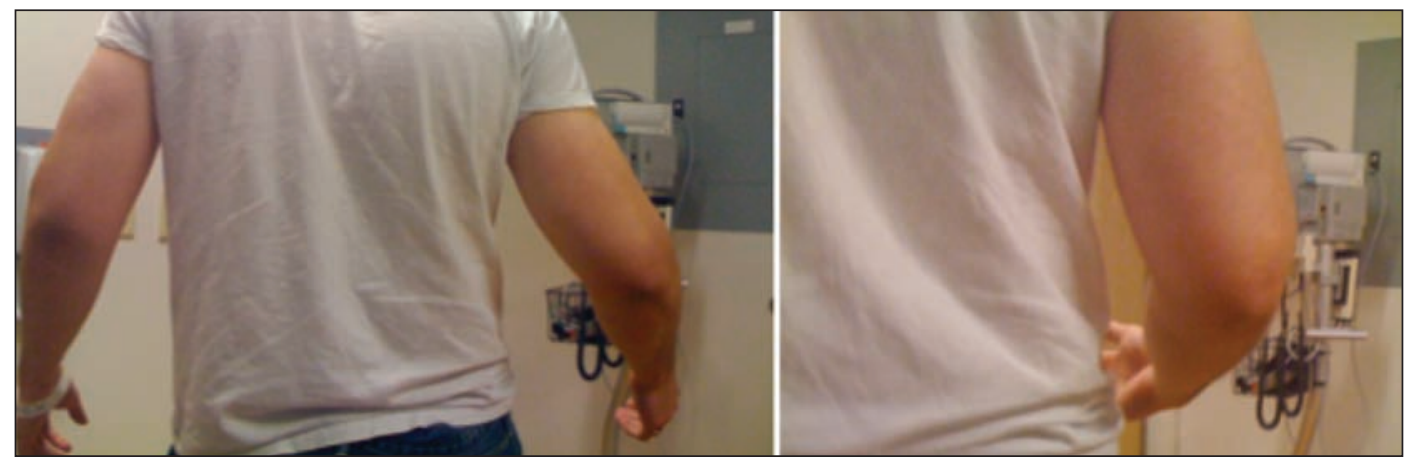

Figure 1: Photos of a 24-year-old man showing swelling of the arms and reduced elbow extension bilaterally, five days after a high-intensity workout.

A 24-year-old man with a history of moderate hypertension, treated with ramipril for two years, presented to the emergency department with progressive swelling of the arms and tingling in his hands. Five days earlier, he had done a short-duration (9 min) high-intensity exercise session that included 84 pull-ups and 84 overhead shoulder presses with 30-lb dumbbells. $\mathrm{He}$ regularly lifted weights and ran as part of his exercise routine, although short-duration high-intensity weight training was something new to him. No traumatic events had occurred between the workout and his visit to the emergency department. Immediately after the high-intensity exercise, his arms felt "tired and sore," but this was not out of keeping with his normal postworkout fatigue. On the day of his visit, bilateral swelling progressed rapidly over three hours, and by the fourth hour, paresthesia had developed. He denied using any supplementation, stimulants, creatine or anabolic steroids. He had no family history of malignant hyperthermia, and he had previously had an uneventful general anesthetic for a minor procedure.

On examination, our patient was alert and afebrile. Both arms were markedly swollen, firm and painful to palpation (Figure 1). His brachial, radial and ulnar pulses were palpable. Laboratory analysis showed a serum creatine kinase level of 84318 (normal 10-234) U/L and myoglobinuria. His calcium, electrolyte, urea and creatinine levels were normal. We diagnosed rhabdomyolysis and started aggressive intravenous fluids. He was dis- charged after two days, with instructions to continue to take oral fluids and refrain from strenuous activity. He had substantial clinical improvement by day 8 , at which time his creatine kinase level was down to $808 \mathrm{U} / \mathrm{L}$. His renal function remained normal.

Exercise-induced rhabdomyolysis is associated with problems ranging from an asymptomatic elevation of creatine kinase levels to severe electrolyte abnormalities, myoglobinuria and renal failure. ${ }^{1-3} \mathrm{~A}$ minimal workup includes testing for serum creatine kinase, calcium and electrolye levels, renal function, quantitative measurement of myoglobin in urine and an electrocardiogram. ${ }^{1}$ It has been suggested that the workup should include testing for malignant hyperthermia. ${ }^{2}$ The most widely accepted treatment for rhabdomyolysis is intravascular volume expansion. ${ }^{4,5}$

Rhabdomyolysis induced by exercise is typically seen in high-endurance athletes involved in marathons, triathlons and super marathons.

\section{References}

1. Patel DR, Gyamfi R, Torres A. Exertional rhabdomyolysis and acute kidney injury. Phys Sportsmed 2009;37.71-9.

2. Capacchione JF, Muldoon SM. The relationship between exertional heat illness, exertional rhabdomyolysis and malignant hyperthermia. Anesth Anaig 2009;109:1065-9.

3. Sayers SP, Clarkson PM. Exercise-induced rhabdomyolysis. Curr Sports Med Rep 2002;1:59-60.

4. Homsi E, Barreiro MF, Orlando JM, et al. Prophylaxis of acute renal failure in patients with rhabdomyolysis. Ren Fail 1997;19: 283-8.

5. Brown CV, Rhee $\mathrm{P}, \mathrm{Chan} \mathrm{L}$, et al. Preventing renal failure in patients with rhabdomyolysis: Do bicarbonate and mannitol make a difference? J Trauma 2004;56:1191-6. 